\title{
On Some Functional Equations Involving Involutions
}

\author{
By \\ Andrzej Mach and Zenon Moszner \\ (Vorgelegt in der Sitzung der math.-nat. Klasse am 22. März 2007 \\ durch das w. M. Ludwig Reich)
}

\begin{abstract}
In this note we present some theorems characterizing solutions of the equation $f(x)=f(\varphi(x))+g(x)$, where $\varphi$ is a given involution, and particulary differentiable solutions of the equation $f(x)=f(1-x)+2 x-1$. The stability of this equation and nonexistence of the extremal points of the set of solutions are proved.
\end{abstract}

Mathematics Subject Classification (2000): 39B72.

Key words: Functional equations, involution, stability, extremal points.

\section{Introduction}

WŁODZIMIERZ WYSOCKI, in connection with the problems of copulas, considers the fixed points of the application $f \mapsto f(1-x)+2 x-1$ in the class $\mathcal{F}$ of functions $f:[0,1] \rightarrow \mathbf{R}$, such that $f(0)=f_{+}^{\prime}(0)=0$, $f(1)=1, f_{-}^{\prime}(1)=2, f^{\prime \prime}(x)>0$, for $\left.x \in\right] 0,1[$ (thus the functions $f$ are the bijections on $[0,1])$. This consideration gives the functional equation

$$
f(x)=f(1-x)+2 x-1
$$

and the generalization of this equation, namely

$$
f(x)=f(\varphi(x))+g(x),
$$


where $\varphi: E \rightarrow E$ is a given involution, this means

$$
\varphi(\varphi(x))=x,
$$

$f, g: E \rightarrow G$ are unknown functions, $E$ is an arbitrary set and $(G,+)$ is an arbitrary group.

In this note we present some theorems characterizing solutions of Eqs. (1) and (2) and particularly, the differentiable solutions of Eq. (1).

It is proved the stability of Eq. (2) and of the equation

$$
g(\varphi(x))+g(x)=0 .
$$

Moreover, we obtain the nonexistence of the extremal points of the set of solutions of (2).

\section{General Case}

\subsection{General Solution}

Let $\varphi: E \rightarrow E$ be an involution.

Definition. We define the equivalence relation $\rho \subset E \times E$ by the following formula

$$
x \rho y \Leftrightarrow y=\varphi(x) \quad \text { or } \quad y=x .
$$

By $E_{1}$ we denote an arbitrary selection of the set $E / \rho$ and let $E_{2}:=E \backslash E_{1}$ and $E_{3}:=E \backslash\left(E_{2} \cup \varphi\left(E_{2}\right)\right)=\{x \in E: \varphi(x)=x\}$.

Theorem 1. The functions $f, g: E \rightarrow G$ are the solutions of $E q$. (2) if and only if

$$
\begin{aligned}
& f(x)= \begin{cases}a(x) & \text { for } x \in E_{1}, \\
a(\varphi(x))+b(x) & \text { for } x \in E_{2},\end{cases} \\
& g(x)=\left\{\begin{array}{lll}
b(x) & \text { for } & x \in E_{2}, \\
-b(\varphi(x)) & \text { for } & x \in \varphi\left(E_{2}\right), \\
0 & \text { for } & x \in E_{3},
\end{array}\right.
\end{aligned}
$$

where $a: E_{1} \rightarrow G, b: E_{2} \rightarrow G$ are the arbitrary functions.

Moreover, the function $g(x)$ given by formula (6) is a solution of Eq. (4).

Easy calculations show that functions defined by (5) and (6) satisfy (2) and the function (6) fulfils (4).

Proof of "only if". We suppose that the functions $f, g$ satisfy Eq. (2). We define $a(x):=f(x)$ for $x \in E_{1}$ and $b(x):=g(x)$ for $x \in E_{2}$. If $x \in E_{2}$ 
then we have by Eq. (2)

$$
f(x)=f(\varphi(x))+g(x)=a(\varphi(x))+b(x) .
$$

This means that the function $f$ has the form (5). If $x \in \varphi\left(E_{2}\right)$ then we have $f(x)=a(x), f(\varphi(x))=a(x)+b(\varphi(x))$ and by Eq. (2)

$$
a(x)=a(x)+b(\varphi(x))+g(x),
$$

so $g(x)=-b(\varphi(x))$. If $x \in E_{3}$ then we have $f(x)=a(x), f(\varphi(x))=$ $a(x)$ and by Eq. (2)

$$
a(x)=a(x)+g(x),
$$

so $g(x)=0$.

It turns out that the general solution of (4) is given by formula (6) only in the case where the group $G$ has no element of the order 2 . More precisely, we have the following result, the proof of which is similar to that of Theorem 1 .

Theorem 2. The function $g$ given by the formula

$$
g(x)= \begin{cases}b(x) & \text { for } x \in E_{2}, \\ -b(\varphi(x)) & \text { for } x \in \varphi\left(E_{2}\right), \\ c(x) & \text { for } x \in E_{3},\end{cases}
$$

where $b: E_{2} \rightarrow G, c: E_{3} \rightarrow G_{1}:=\{\alpha \in G: 2 \alpha=0\}$ are the arbitrary functions, is the general solution of $E q$. (4).

The proof of the below presented theorem is evident.

Theorem 3. The general solution of Eq. (3) has the form

$$
\varphi(x)=\left\{\begin{array}{lll}
\psi(x) & \text { for } & x \in Z_{2}, \\
\psi^{-1}(x) & \text { for } & x \in \psi\left(Z_{2}\right), \\
x & \text { for } & x \in E \backslash\left(Z_{2} \cup \psi\left(Z_{2}\right)\right),
\end{array}\right.
$$

where $Z_{2} \subset E$ is such that $\operatorname{card} Z_{2} \leq \operatorname{card}\left(E \backslash Z_{2}\right)$ and $\psi: Z_{2} \rightarrow E \backslash Z_{2}$ is an arbitrary injection. Thus the general solution of the system of Eqs. (2) and (3) is of the form (7),

$$
\begin{aligned}
& f(x)= \begin{cases}a(x) & \text { for } \quad x \in Z_{1}:=E \backslash Z_{2}, \\
a(\psi(x))+b(x) & \text { for } \quad x \in Z_{2},\end{cases} \\
& g(x)= \begin{cases}b(x) & \text { for } \quad x \in Z_{2}, \\
-b\left(\psi^{-1}(x)\right) & \text { for } \quad x \in \psi\left(Z_{2}\right), \\
0 & \text { for } \quad x \in E \backslash\left(Z_{2} \cup \psi\left(Z_{2}\right)\right),\end{cases}
\end{aligned}
$$


where $Z_{2}$ and $\psi$ are as above and $a: Z_{1} \rightarrow G, b: Z_{2} \rightarrow G$ are the arbitrary functions.

In the next result (2) is considered as an equation with an unknown function $f$ and a given function $g$.

Theorem 4. $\left(1^{0}\right)$ Equation (2) has a solution if and only if $g$ satisfies (4).

Assume that $g, g_{1}: E \rightarrow G$ satisfy (4).

$\left(2^{0}\right)$ If $f_{1}: E \rightarrow G$ is a solution of $(2)$, and $\Phi: G \rightarrow G$ is an even function, then the function $f: E \rightarrow G$ given by

$$
f=\Phi \circ g_{1}+f_{1}
$$

satisfies (2).

$\left(3^{0}\right)$ If $g_{1}$ is invertible then the general solution $f: E \rightarrow G$ of (2) can be obtained in the following way. Fix a solution $f_{1}: E \rightarrow G$ of (2), choose an even function $\Phi: g_{1}(E) \rightarrow G$ and put (8).

$\left(4^{0}\right)$ The general solution of (2) is a sum of general solutions of the equation

$$
m(\varphi(x))=m(x)
$$

and of the particular solution of (2). The general solution of Eq. (9), for arbitrary sets $E$ and $G$, is of the form

$$
m(x)=\left\{\begin{array}{lll}
d(x) & \text { for } & x \in E_{1}, \\
d(\varphi(x)) & \text { for } & x \in E_{2},
\end{array}\right.
$$

where $d: E_{1} \rightarrow G$ is an arbitrary function.

Proof. The proof in the cases $\left(1^{0}\right),\left(2^{0}\right)$ and $\left(4^{0}\right)$ is obvious. In case $\left(3^{0}\right)$, the function $\Phi(u)=f\left[g_{1}^{-1}(u)\right]-f_{1}\left[g_{1}^{-1}(u)\right]$, for some solutions $f$ and $f_{1}$ of (2), is even. Indeed, since $g_{1}(\varphi(x))=-g_{1}(x)$, then $\varphi\left[g_{1}^{-1}(u)\right]=g_{1}^{-1}(-u)$, the set $g_{1}(E)$ is symmetric in relation to 0 (if $a=g_{1}(x)$ then $-a=g_{1}(\varphi(x))$ ) and

$$
\begin{aligned}
\Phi(-u) & =f\left[g_{1}^{-1}(-u)\right]-f_{1}\left[g_{1}^{-1}(-u)\right]=f\left\{\varphi\left[g_{1}^{-1}(u)\right]\right\}-f_{1}\left\{\varphi\left[g_{1}^{-1}(u)\right]\right\} \\
& =f\left[g_{1}^{-1}(u)\right]-g\left(g_{1}^{-1}(u)\right)-\left\{f_{1}\left[g_{1}^{-1}(u)\right]-g\left(g_{1}^{-1}(u)\right)\right\} \\
& =\Phi(u) \quad \text { for } \quad u \in g_{1}(E) .
\end{aligned}
$$

Remark 1. Every even function $\Psi: g_{1}(E) \rightarrow G$ is obviously extended to the even function on $G$ since $g_{1}(E)$ is symmetric in relation to 0 , there it is possible to use in $\left(3^{0}\right)$ for $\Phi$ the even function on $G$ (not only on $g_{1}(E)$ ). 
Remark 2. The invertible solution of (4) cannot exist, e.g., for $E=\mathbf{R},(G,+)=(\mathbf{R},+), \varphi(x)=x$. This solution exists if and only if $\operatorname{card} E_{2} \leq \operatorname{card}\left(G \backslash G_{1}\right) / R$ and $\operatorname{card} E_{3} \leq \operatorname{card} G_{1}$, where for $\alpha, \beta \in$ $G \backslash G_{1}: \alpha R \beta \Leftrightarrow(\beta=-\alpha$ or $\beta=\alpha)$ (see Theorem 2). The invertible solutions of (4) are determined exactly to an invertible, odd function. For two invertible solutions $g_{1}$ and $g_{2}$ of (4) we have

$$
g_{2}\left[g_{1}^{-1}(-u)\right]=g_{2}\left[\varphi\left(g_{1}^{-1}(u)\right)\right]=-g_{2}\left[g_{1}^{-1}(u)\right] \quad \text { for } \quad u \in g_{1}(E),
$$

thus $g_{2}=\Psi\left(g_{1}\right)$, where $\Psi: g_{1}(E) \rightarrow G$ is invertible and odd. Inversely, if $g_{1}$ is an invertible solution of (4), then $g_{2}=\Psi\left(g_{1}\right)$ is the same solution, where $\Psi: g_{1}(E) \rightarrow G$ is an arbitrary, invertible and odd function.

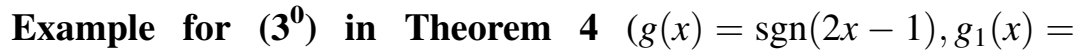
$2 x-1)$. For the equation

$$
f(x)=f(1-x)+\operatorname{sgn}(2 x-1),
$$

where $f: \mathbf{R} \rightarrow \mathbf{R}$ and sgn $0:=0, f_{1}(x)=1$ for $x \geq \frac{1}{2}$ and $f_{1}(x)=0$ for $x<\frac{1}{2}$ is a particular solution, $\Phi(2 x-1)+f_{1}(x)$ for the arbitrary even function $\Phi: \mathbf{R} \rightarrow \mathbf{R}$ is a general solution and $\Phi[\operatorname{sgn}(2 x-1)]+f_{1}(x)$ is a solution but not the general solution. Indeed, this function is for $x>\frac{1}{2}$ equal to $\Phi(1)+1$ and the function $(2 x-1)^{2}+f_{1}(x)$ is a solution of our equation and it is not constant for $x>\frac{1}{2}$. Therefore, the assumption that $g_{1}$ is invertible is essential in $\left(3^{0}\right)$ of Theorem 4 .

\subsection{Stability}

We will prove that the system of Eqs. (2) and (4), Eq. (2) and Eq. (4), are stables for some metrics.

Theorem 5. $\left(1^{0}\right)$ Let us suppose that $\rho$ is the left translation-invariant metric in the group $(G,+)$, this means $\rho(u, v)=\rho(t+u, t+v)$, for $u, v, t \in G$. For every $\varepsilon>0$ and for every functions $a: E \rightarrow G$, $b: E \rightarrow G$ such that

$$
\begin{aligned}
\forall x \in E: & \rho(a(\varphi(x))+b(x), a(x)) \\
\forall x \in E: & \rho(b(\varphi(x))+b(x), 0) \leq \varepsilon,
\end{aligned}
$$

there exists a solution $f: E \rightarrow G, g: E \rightarrow G$ of the system of Eqs. (2) and (4) such that

$$
\forall x \in E: \quad \rho(f(x), a(x)) \leq \varepsilon
$$

and

$$
\forall x \in E: \quad \rho(g(x), b(x)) \leq \varepsilon .
$$


$\left(2^{0}\right)$ If the metric $\rho$ is the left and right translation invariant and only the inequality (10) is true, then (12) is satisfied and we have (13) with $2 \varepsilon$ in place of $\varepsilon$.

$\left(3^{0}\right)$ If the metric $\rho$ is the left translation invariant, $2 \rho(u, 0) \leq$ $\rho(2 u, 0)$, for $u \in G$ and only (11) is true, then (13) is satisfied.

Proof. Functions $f, g$ given by (5), (6) satisfy (2) and (4), by Theorem 1. Hence:

If $x \in E_{2}$, then $\rho[f(x), a(x)]=\rho[a(\varphi(x))+b(x), a(x)]$ and $\rho[g(x)$, $b(x)]=0$.

If $x \in E_{3}, \quad$ then $\quad \rho[f(x), a(x)]=0, \quad \rho[g(x), b(x)]=\rho[0, b(x)]=$ $\rho[a(\varphi(x)), a(\varphi(x))+b(x)]=\rho[a(x), a(\varphi(x))+b(x)]$ and $\rho[0, b(x)] \leq$ $\frac{1}{2} \rho[0,2 b(x)]$ in case $\left(3^{0}\right)$.

If $x \in \varphi\left(E_{2}\right)$, then $\rho[f(x), a(x)]=0$ and $\rho[g(x), b(x)]=\rho[-b(\varphi(x))$, $b(x)]=\rho[0, b(\varphi(x))+b(x)]=\rho[a(x), a(x)+b(\varphi(x))+b(x)] \leq \rho[a(x)$, $a(\varphi(x))+b(x)]+\rho[a(\varphi(x)), a(x)+b(\varphi(x))]$.

Remark 3. The equation

$$
g(x)+g(x)=2 g(x)=0 \quad(\varphi(x)=x)
$$

is not stable for some metric.

Let be

$$
\alpha(x)= \begin{cases}x & \text { for } \quad x \in Z_{1}:=\{2 /(2 n+1): n=1,2, \ldots\}, \\ 1+x & \text { for } \quad x \in Z_{2}:=\{1 /(2 n+1): n=1,2, \ldots\}, \\ \beta(x) & \text { for } \quad x \in Z_{3}:=\mathbf{R} \backslash\left(Z_{1} \cup Z_{2}\right),\end{cases}
$$

where $\beta: Z_{3} \rightarrow \mathbf{R} \backslash\left[Z_{1} \cup\left(Z_{2}+1\right)\right]$ is a bijection, such that $\beta(0)=0$. Let $\rho(a, b):=|\alpha(a)-\alpha(b)|$ be the metric in $\mathbf{R}$. Let $(G,+)=(\mathbf{R},+)$ and $E=\{0\}$. Eq. (14) is not stable with respect to the metric above. Indeed, for $\varepsilon=1$ and $\delta>0$, if $2 /(2 n+1) \leq \delta$ and $k(0)=1 /$ $(2 n+1)$, then

$$
\rho[2 k(0), 0]=|\alpha(2 /(2 n+1))-\alpha(0)|=2 /(2 n+1) \leq \delta
$$

and

$$
\rho[k(0), 0]=|\alpha(1 /(2 n+1))-\alpha(0)|=1+1 /(2 n+1)>1 .
$$

Attention. Here the equation $2 g(x)=0$ is not stable and the equivalent equation $g(x)=0$ is evidently stable.

Remark 4. The system of Eqs. (2), (3), (4) is not stable for some metrics in $E$ and $G$, since the equation of involution is not stable for some translation-invariant metric $\rho^{*}$ in $E$, i.e., the condition below 
(the analogon of the Ulam-Hyers stability of the equation of homomorphism in [1]) is not satisfied:

For every $\varepsilon>0$ there exists a $\delta>0$ such that for every $\psi: E \rightarrow E$ if $\rho^{*}(\psi(\psi(x)), x) \leq \delta$ for $x \in E$, then there exists an involution $\varphi$ such that $\rho^{*}(\varphi(x), \psi(x)) \leq \varepsilon$ for $x \in E$.

Indeed, if $E=\mathbf{R}$ with natural metric, then for arbitrary $\delta>0$ and $n$ fixed such that $1 / n \leq \delta$ and $n>3$, the function $\psi: E \rightarrow E$ such that $\psi(0)=\psi(1 / n)=1, \psi(1)=1 / n, \psi(\alpha)=\alpha$, for $\alpha \in E \backslash$ $\{0,1,1 / n\}=: E^{*}$, satisfies $|\psi(\psi(x))-x| \leq \delta$, for $x \in E$. We suppose that there exists an involution $\varphi$ such that $|\bar{\psi}(x)-\varphi(x)| \leq \frac{1}{3}$, for $x \in E$. Then $|\psi(0)-\varphi(0)|=|1-\varphi(0)| \leq \frac{1}{3}$, thus $\quad \frac{2}{3} \leq a:=\varphi(0) \leq \frac{4}{3}$. We have $|\psi(a)-\varphi(a)|=|\psi(a)-0|=|\psi(a)| \leq \frac{1}{3}$, thus $a=0$ or $a=1 / n$ is impossible. If $a \in E^{*}$, then $|a| \leq \frac{1}{3}$, thus a contradiction. Therefore, $\varphi(0)=a=1$. We have $|\psi(1 / n)-\varphi(1 / n)|=$ $|1-\varphi(1 / n)| \leq \frac{1}{3}$, thus $\frac{2}{3} \leq b:=\varphi(1 / n) \leq \frac{4}{3}$ and $|\psi(b)-(1 / n)| \leq \frac{1}{3}$. For $b \in E^{*}:|b-(1 / n)| \leq \frac{1}{3}$, thus $b \leq(1 / n)+\frac{1}{3}<\frac{2}{3}$, we obtain a contradiction. For $b=0$ or $b=1 / n$ the contradiction is evident. Therefore, $\varphi(1 / n)=b=1$. A contradiction, since $\varphi$, as involution, is injective.

If $(G,+)$ is the group with arbitrary metric $\rho$, then the triple $(h(x), k(x), \psi(x))=(0,0, \psi(x))$ satisfies the inequalities $\rho[h(\psi(x))+$ $k(x), h(x)] \leq \delta, \rho[k(\psi(x))+k(x), 0] \leq \delta,|\psi(\psi(x))-x| \leq \delta$, for $x \in E$, and the solution $(f, g, \varphi)$ of the system: (2), (4), (3) such that $|\psi(x)-\varphi(x)| \leq \frac{1}{3}$, for $x \in E$, does not exist.

If the metric is $0-1(\rho(x, x)=0$ and $\rho(x, y)=1$ for $x \neq y)$ then every functional equation (or system) is stable (it is sufficient to put arbitrary positive $\delta<1$ for all $\varepsilon>0$ ).

\section{Particular Case. Differentiable Solutions}

By Theorem 4 we have the following corollaries.

Corollary 1. The function $f: \mathbf{R} \rightarrow \mathbf{R}$ given by the formula

$$
f(x)=x^{2}+\Phi(2 x-1),
$$

where $\Phi: \mathbf{R} \rightarrow \mathbf{R}$ is an arbitrary even function, is the general solution of the functional equation (1) without any regularity condition.

The differentiable function $f: \mathbf{R} \rightarrow \mathbf{R}$ is the solution of the functional equation (1), for which $f^{\prime}(0)=0$ and $f^{\prime}(1)=2$, if and only if there exists a differentiable and even function $\Phi: \mathbf{R} \rightarrow \mathbf{R}$, such that $\Phi^{\prime}(-1)=\Phi^{\prime}(1)=0$ and $(15)$ is satisfied.

Corollary 2. The function $f:[0,1] \rightarrow \mathbf{R}$ belonging to the class $\mathcal{F}$ is the solution of the functional equation (1), if and only if there exists a 
twice differentiable and even function $\Phi$ defined on interval $[-1,+1]$, such that

$$
\begin{array}{cr}
-\frac{1}{4} x^{2}-\frac{1}{2} x-\frac{1}{4} \leq \Phi(x) \leq-\frac{1}{4} x^{2}-\frac{1}{2} x+\frac{3}{4} & \text { for all } x \in]-1,+1[, \\
x+2 \Phi^{\prime}(x)+1>0 & \text { for all } x \in]-1,+1[, \\
1+2 \Phi^{\prime \prime}(x)>0, & \text { for all } x \in]-1,+1[, \\
\Phi(-1)=\Phi(1)=0, & \Phi^{\prime}(-1)=\Phi^{\prime}(1)=0
\end{array}
$$

and (15) is satisfied.

The next result is a consequence of Theorem 1 .

Corollary 3. All solutions of Eq. (1) in the class $\mathcal{F}$ can be obtained by the following way. Let us take an arbitrary function $h:\left[0, \frac{1}{2}\right] \rightarrow$ $[0,1]$ satisfying

$$
\begin{gathered}
h(0)=h_{+}^{\prime}(0)=0, \quad h_{-}^{\prime}\left(\frac{1}{2}\right)=1, \\
\left.\left.h^{\prime \prime}(x)>0, \quad \text { for all } \quad x \in\right] 0, \frac{1}{2}\right] .
\end{gathered}
$$

We put

$$
f(x)= \begin{cases}h(x) & \text { for } x \in\left[0, \frac{1}{2}\right], \\ h(1-x)+2 x-1 & \text { for } \left.x \in] \frac{1}{2}, 1\right] .\end{cases}
$$

Theorem 6. Let $f: \mathbf{R} \rightarrow \mathbf{R}$ be a differentiable function, for which there exists $f^{\prime \prime}(0)$. Moreover, let $g: \mathbf{R} \rightarrow \mathbf{R}$ be a differentiable function, for which $g^{\prime}(x) \neq 0, x \in \mathbf{R}$ and $g^{\prime}$ is continuous at 0 . If the functions $f, g$ satisfy the following system of functional and differential equations

$$
\begin{aligned}
f(x) & =f(1-x)+g(x), \\
{\left[g^{\prime}(x)\right]^{2} \cdot f^{\prime}\left(x^{2}\right) } & =g^{\prime}\left(x^{2}\right) \cdot\left[f^{\prime}(x)\right]^{2},
\end{aligned}
$$

then $f(x)=\int x g^{\prime}(x) d x$.

Proof. By Eq. (16) we get

$$
f^{\prime}(x)+f^{\prime}(1-x)=g^{\prime}(x)
$$

and by Eq. (17) we have $f^{\prime}(0)=0$ or $f^{\prime}(0)=g^{\prime}(0)$.

Let us define $h(x):=f^{\prime}(x) / g^{\prime}(x)$. Since (16) implies $g^{\prime}(x)=$ $g^{\prime}(1-x)$, then by (18) we have

$$
h(x)+h(1-x)=1
$$

and $h(0)=0$ or $h(0)=1$. 
One can observe easily that Eq. (17) implies

$$
h\left(x^{2}\right)=h^{2}(x),
$$

therefore $h^{2}(-x)=h^{2}(x)$, so

$$
h(x)=-h(-x) \quad \text { or } \quad h(x)=h(-x), \quad \text { for all } \quad x \in \mathbf{R} .
$$

Putting $x=\frac{1}{2}$ in Eq. (19) we get $h\left(\frac{1}{2}\right)=\frac{1}{2}$. Putting $x=\frac{1}{2}$ in Eq. (20) we get $h\left(\frac{1}{4}\right)=\frac{1}{4}$ and generally

$$
h\left(1 / 2^{2^{n}}\right)=1 / 2^{2^{n}},
$$

for every $n \in \mathbf{N}$, thus $h(0)=0$. Remark that since

$$
h_{+}^{\prime}(0)=\lim _{x \rightarrow 0^{+}} \frac{h(x)}{x}=\lim _{x \rightarrow 0^{+}} \frac{\frac{f^{\prime}(x)}{x}}{g^{\prime}(x)}=\frac{f_{+}^{\prime \prime}(0)}{g_{+}^{\prime}(0)},
$$

then $h_{+}^{\prime}(0)$ exists. Therefore (21) implies that $h(0)=0$ and $h_{+}^{\prime}(0)=1$.

It is easy to see that there exists $\varepsilon>0$ such that $h(x)>0$, for $x \in] 0,+\varepsilon[$ and $h(x)<0$, for $x \in]-\varepsilon, 0[$, thus we have $h(x)=-h(-x)$, for every $x \in]-\varepsilon,+\varepsilon[$.

Indeed, on the contrary we get easily the contradiction with the supposition that there exists $f^{\prime \prime}(0)\left(=g^{\prime}(0) \cdot h^{\prime}(0)\right)$.

We will prove that the function $h$ is the odd function, so

$$
h(x)=-h(-x), \quad \text { for every } \quad x \in \mathbf{R} .
$$

Indeed, on the contrary, let us put $x_{0}:=\inf \{x \geq \varepsilon: h(x)=h(-x)\}$. One can observe easily that $h\left(x_{0}\right)=0$ necessarily. Putting in Eq. (19) $x:=x_{0}$ we get $h\left(1-x_{0}\right)=1$. If $1-x_{0} \leq 0$, then $0 \leq x_{0}-1 \leq x_{0}$ and $1=h\left(1-x_{0}\right)=-h\left(x_{0}-1\right) \leq 0$ in view of (19). This implies $1-x_{0}>0$, so $x_{0}<1$. By Eq. (20) we get $h\left(x_{0}^{2}\right)=h^{2}\left(x_{0}\right)=0$, therefore $h\left(x_{0}^{2 n}\right)=0$, for every $n \in \mathbf{N}$ and $\lim _{n \rightarrow \infty} x_{0}^{2 n}=0$. This is the contradiction with $h_{+}^{\prime}(0)=1$, then we have (22).

Replacing $x$ by $x+1$ in Eq. (19), we get $h(x+1)+h(-x)=1$, so $h(x+1)=-h(-x)+1$, therefore

$$
h(x+1)=h(x)+1 .
$$

By the result of VOLKMANN (see [2]), since the function $h$ satisfies Eqs. (23) and (20), then $h(x)=x$. This implies $f^{\prime}=x \cdot g^{\prime}(x)$ and $f(x)=\int x g^{\prime}(x) d x$. 
Corollary 4. If $f: \mathbf{R} \rightarrow \mathbf{R}$ is a differentiable solution of (1), for which there exists $f^{\prime \prime}(0)$ and

$$
2 f^{\prime}\left(x^{2}\right)=\left[f^{\prime}(x)\right]^{2}
$$

then $f(x)=x^{2}+c$, where $c \in \mathbf{R}$.

\section{Convexity. Extremal Points}

$\left(1^{0}\right)$ If the group $(G,+)$ is Abelian and divisible by 2 , then the set of solutions $(f, g)$ of $(2)$ is evidently convex and it has no extremal points. Indeed, for

$$
f_{1}(x)= \begin{cases}a_{1}(x) & \text { for } \quad x \in E_{1}, \\ a_{1}(\varphi(x)) & \text { for } \quad x \in E_{2},\end{cases}
$$

where $a_{1}: E_{1} \rightarrow G$ and $a_{1}(x) \neq 0$ and for the solution $(f, g)$ of (2), the pairs $\left(f+f_{1}, g\right)$ and $\left(f-f_{1}, g\right)$ are the solutions of $(2)$,

$$
(f, g)=\frac{\left(f+f_{1}, g\right)+\left(f-f_{1}, g\right)}{2}
$$

and $\left(f+f_{1}, g\right) \neq(f, g) \neq\left(f-f_{1}, g\right)$.

Analogously the set of solutions of Eq. (2) with $f$ unknown and $\varphi$ and $g$ given and the set of solutions of (4) are convex and have no extremal points.

$\left(2^{0}\right)$ The set $\mathcal{F} \cap C^{2}[0,1]$ and the set $\mathcal{F}_{1}$ of solutions of (1) of class $C^{2}[0,1] \cap \mathcal{F}$ are convex and they have no extremal points. Indeed, let be for $f \in \mathcal{F} \cap C^{2}[0,1]$

$$
l(x)= \begin{cases}0 & \text { for } \quad x \in\left[0, \frac{1}{3}\right], \\ \frac{m}{288 \pi^{2}} \sin [4 \pi(3 x-1)]-\frac{m}{24 \pi}\left(x-\frac{1}{3}\right) & \text { for } \left.\quad x \in] \frac{1}{3}, \frac{1}{2}\right],\end{cases}
$$

where $m=\min _{\left[\frac{1}{3}, \frac{1}{2}\right]} f^{\prime \prime}(x)$, and

$$
f_{1}(x)= \begin{cases}l(x) & \text { for } \quad x \in\left[0, \frac{1}{2}\right] \\ l(1-x) & \text { for } \left.\quad x \in] \frac{1}{2}, 1\right] .\end{cases}
$$

Then $f \pm f_{1} \in \mathcal{F} \cap C^{2}[0,1]$ and

$$
\frac{\left(f+f_{1}\right)+\left(f-f_{1}\right)}{2}=f \quad \text { and } \quad f+f_{1} \neq f \neq f-f_{1} .
$$

If $f \in \mathcal{F}_{1}$, then $f \pm f_{1} \in \mathcal{F}_{1}$ too. 
Remark 5. The set $\mathcal{F}_{0}$ of continuous bijections $f$ on $[0,1]$ such that $f(0)=0$ or equivalently of increasing bijections (respectively: such that $f(0)=1$ or equivalently of decreasing bijections), is convex and has no extremal points. To be sufficient put, for $f \in \mathcal{F}_{0}$ and $x_{0}$ such that $f\left(x_{0}\right)=\frac{1}{2}$

$$
\begin{array}{r}
f_{1}(x)=\left\{\begin{array}{lll}
\frac{1}{2} f(x) & \text { for } & x \in\left[0, x_{0}\right], \\
-\frac{1}{2} f(x)+\frac{1}{2} & \text { for } & \left.x \in] x_{0}, 1\right],
\end{array}\right. \\
\text { (respectively: } f_{1}(x)=\left\{\begin{array}{lll}
-\frac{1}{2} f(x)+\frac{1}{2} & \text { for } & x \in\left[0, x_{0}\right], \\
\frac{1}{2} f(x) & \text { for } & \left.x \in] x_{0}, 1\right],
\end{array}\right),
\end{array}
$$

so as to have $f \pm f_{1} \in \mathcal{F}_{0}$ and (24).

The set of bijections on $[0,1]$ (continuous too) is not convex $\left((f+(1-f)) / 2=\frac{1}{2}\right.$ for every bijection $\left.f\right)$.

\section{Acknowledgement}

We are thankful to the referee for all remarks and suggestions.

\section{References}

[1] Hyers, D. H. (1941) On the stability of the linear functional equation. Proc. Natl. Acad. Sci. USA 27: 222-224

[2] Volkmann, P. (1983) Caractérisation de la fonction $f(x)=x$ par un systéme de deux équations fonctionnelles. Compt. Rend. Math. Acad. Sci. 5(1): 27-28

Authors' addresses: Dr. Andrzej Mach, Institute of Mathematics, Jan Kochanowski University, Świętokrzyska 15, 25-406 Kielce, Poland. E-Mail: amach@pu.kielce.pl; Prof. Dr. Dr. h.c. Zenon Moszner, Institute of Mathematics, Akademia Pedagogiczna, Podchorążych 2, 30-084 Kraków, Poland. E-Mail: zmoszner@ap.krakow.pl. 